\title{
NO EROSION CRITERION FOR GRANULAR FILTERS BASED ON CONSTRICTION SIZE - AN EXPERIMENTAL STUDY
}

\author{
Mohan Das C, Dr. M S Girish \\ College of Engineering Trivandrum, Kerala, India \\ Vishnu V K \\ Jawaharlal College of \& Engineering Technology, \\ Palakkad, Kerala, India
}

\begin{abstract}
Filters are provided in embankments in order to arrest the eroded soil particles that are carried by the seepage water. Current design method of graded filters was developed by Terzaghi which is based on the particle size distribution of the filter material and the base material. This paper presents an attempt to develop filter design criteria in relation to constriction size in gravel filters. It is an alternative approach from the conventional filter design criteria of Terzaghi, which is based on the particle size distribution of the filter media and base material. In his study a criterion for filter to prevent erosion is proposed based on the constriction size distribution of the filter media. The proposed constriction size-based criterion, $C_{10} / d_{95} \leq 2$ directly compares the controlling constriction size $\left(C_{10}\right)$ with the specific representative base size $\left(d_{95}\right)$ defines the effectiveness of filters, hence it is a more effective method compared to the Terzaghi criterion $\left(D_{15} / d_{85}<5\right)$ which is based purely on particle size. This ratio explains the behavior of filters more clearly and is proved by a number of tests on various base-filter combinations. When compared to Terzaghi criteria, constriction size distribution criterion proves to be more effective
\end{abstract}

Keywords: Graded filters, Earth dams, Constriction size, No erosion criterion

Cite this Article: Mohan Das C, Dr. M S Girish and Vishnu V K, No Erosion Criterion for Granular Filters Based on Constriction Size - An Experimental Study, International Journal of Advanced Research in Engineering and Technology, 10 (2), 2019, pp 374380.

http://iaeme.com/Home/issue/IJARET?Volume $=10 \&$ Issue $=2$

\section{INTRODUCTION}

One of the important functions of filters in embankment dams is to prevent the erosion of fine particles of the base material carried by the seepage water. The filters are placed at critical locations of the embankment so as to make it effective. Failure of a filter can happen in two ways, either it fails to trap the eroding fine particles or it gets clogged by the eroding material. 
Nearly $50 \%$ of the dam failures were attributed to defective filters [6]. The criteria for design of filters was developed by Karl Terzaghi which is still used by engineers. The existing Terzaghi's criterion of filter design are based on the ratio $\mathrm{D}_{15} / \mathrm{d}_{85} \leq 5$ (Here $\mathrm{D}_{15}$ denotes that $15 \%$ of the particles are finer than diameter D of filter material, and $\mathrm{d}_{85}$ denotes that $85 \%$ of the particles are finer than the diameter $d$ of base material) $[2,4]$ for retention of particles. Terzaghi's criterion has a serious drawback that it will not give a unique particle size distribution suitable for a filter. A sand gravel filter that satisfies Terzaghi criterion can have different particle size distributions making it difficult to choose the most suitable one. Recently new approaches for determining the filter criteria have been proposed. The constriction size distribution of the filter medium is proposed as a more rational approach for determining the filter criteria [4]. M A Lone et.al proposed an approach for filter design based on packing pattern of nonuniform size particles.[4]. Investigations by Indraratna et.al [1] developed a criterion based on the constriction size distribution of filters and surface area of the base materials for effective filters as $\mathrm{C}_{35} / \mathrm{d}_{85 \mathrm{SA}}<1$ where $\mathrm{C}_{35}$ being the constriction size corresponding to $35 \%$ probability and $\mathrm{d}_{85 \mathrm{SA}}$, the percentage finer surface area of the base material . This criterion was found to be applicable for uniformly and widely distributed base materials. However, the computation of particle surface area distribution makes the expression unfavorable for practical purposes. Also numerical studies on the migration of fine particles through the filter medium have been reported [7, 8]. For earth dams the base materials will be usually of uniformly graded or moderately uniformly graded with uniformity coefficient $\mathrm{C}_{\mathrm{u}}<5$. This paper presents a study to develop a retention criterion for filter design in relation to constriction size distribution in filters and the particle size distribution of the base soil for uniformly graded base materials. By establishing the relation, a unique graded filter can be selected more easily for a given base material.

\section{METHODOLOGY}

\subsection{Conceptual model for particle packing}

It is assumed that particle have spherical shape, and also irrespective of the initial packing of spheres, a slight disturbance causes the spheres to rearrange themselves in such a way that three particles come together in a plane to form an interparticle pore. If the third dimension is considered, the gap formed by three spheres is occupied by the fourth sphere in the second plane. Hence, this configuration seems to be the most logical for studying the engineering behavior of particulate medium (such as sand, gravel, boulders, etc.) The arrangement is shown in Fig. 1.[4]

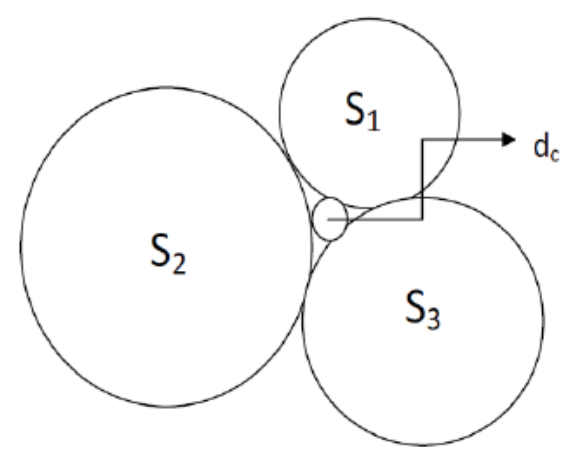

Figure 1. Unit assembly comprised of three primary spheres and one filler sphere. 


\subsection{Constriction size distribution of filter medium}

The constriction size distribution (CSD) can be derived from the particle size distribution of filter medium for the densest medium [5]. Three mutually tangent spheres with diameters $d_{i}, d_{j}, d_{k}$ form a constriction of $d_{c}$ (Fig .1). The diameter of the constriction $d_{c}$ is given by Descartes' theorem

$$
\frac{1}{d_{c}}=\left(\frac{1}{d_{i}}\right)+\left(\frac{1}{d_{j}}\right)+\left(\frac{1}{d_{k}}\right) \pm 2 \sqrt{\left(\frac{1}{d_{i^{*} d_{j}}}\right)+\left(\frac{1}{d_{j^{*}} d_{k}}\right)+\left(\frac{1}{d_{k} * d_{i}}\right)}
$$

If the probability of occurance of diameter $\mathrm{di}, \mathrm{dj}_{\mathrm{j}}$ and $\mathrm{d}_{\mathrm{k}}$ are respectively $p_{i,} p_{j}$ and $p_{k}$ then the probability of diameter of constriction $\mathrm{P}_{\mathrm{c}}$ is

$$
\mathrm{P}_{\mathrm{c}}=\frac{3 !}{r_{i} ! r_{j} ! r_{k} !} p_{i}^{r_{i}} p_{j}^{r_{j}} p_{k}^{r_{k}}
$$

where $r_{i}, r_{j}$ and $r_{k}$ denote the number of occurrences of $d_{i}, d_{j}$ and $d_{k}$ in the group of three particles $\left(r_{i}+r_{j}+r_{k}=3\right)$ respectively [5]. The probabilities $p_{i}, p_{j}$ and $p_{k}$ can obtained obtained from the particle size distribution curve by discretizing it (Fig.2) .Considering all possible combinations the complete CSD is obtained [5].

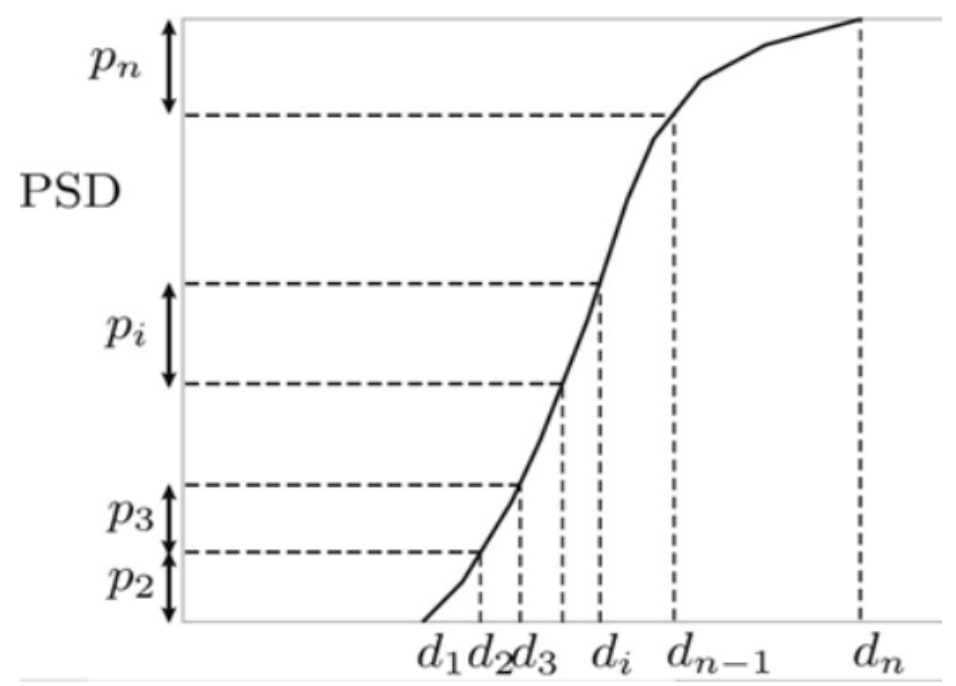

Figure 2 A discretized particle size distribution $\left(\mathrm{p}_{1}=0\right)[5]$

\section{EXPERIMENTAL PROGRAMME}

\subsection{Experimental setup}

The experiment was conducted in the hydraulics laboratory of College of Engineering Trivandrum. Experimental setup consists of a cylinder of diameter $20 \mathrm{~cm}$ and length $60 \mathrm{~cm}$, a perforated plate is fixed at bottom to prevent the movement of filter materials. $30 \mathrm{~cm}$ height of filter material, $10 \mathrm{~cm}$ height of base material and $10 \mathrm{~cm}$ constant water height was maintained in the cylinder as shown in Fig.3. 


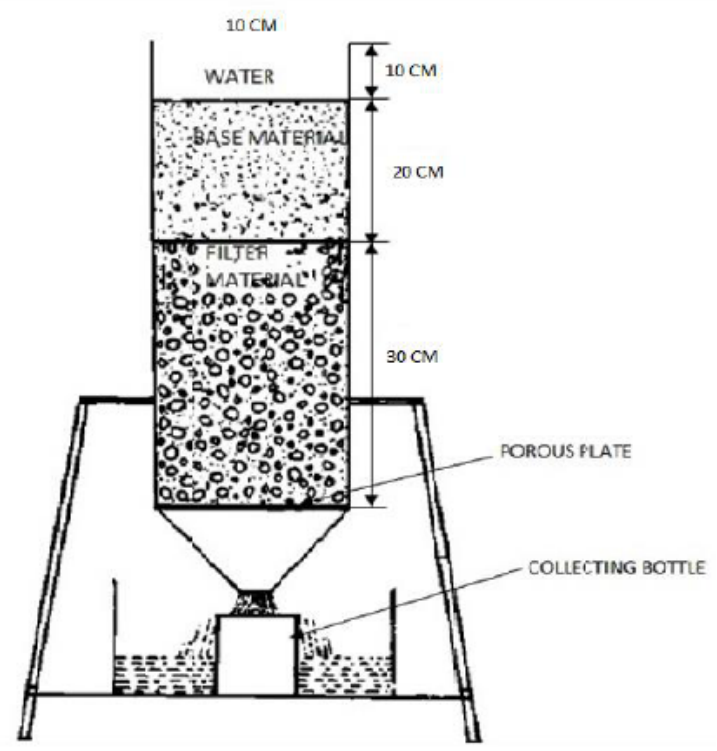

Figure 3 Experimental setup

\subsection{Materials}

Sieve the base and filter materials and draw the gradation curve for different samples collected. For the base materials, sands of various gradations were used and their particles size ranged from 0.075 to $4.75 \mathrm{~mm}$. For the filter material, gravel of various gradations with particle size greater than $4.75 \mathrm{~mm}$ were used.

\subsection{Test procedure}

Place the filter materials over the perforated plate at the bottom of cylinder for a thickness of $30 \mathrm{~cm}$ and compacted. Then base materials were placed over the filter material. The thickness of base materials is taken as $20 \mathrm{~cm}$. A constant head of $10 \mathrm{~cm}$ water is maintained at the top of the base material, with an overflow arrangement. Depending on the type of base materials and filter materials the amount of particles getting eroded from the base materials vary and were collected using a bottle placed below the cylinder. The size and amount of base materials eroded were estimated using a image processing software when eroded particles were very less in quantity. If substantial erosion takes place, quantity is measured by weight. One of the options of the image processing software is the determination of particle size distribution. For finding the particle size and quantity, a photograph of eroded particles were taken and given as input to the software which directly yielded the number of particles present in the sample. The image must be clear and each particle should be seen separately.

The test procedures were repeated by changing the base and filer materials.

\subsection{Constriction Size Distribution Curve for Filter Materials}

From the combination of three mutually perpendicular particles in filter material, constriction size made by three particles was calculated using Descarte's theorem and the respective probability of occurrence of constriction was calculated using equation 2 . Constriction size distribution curve (CSD) was plotted using constriction size and their respective probabilities.

Using different combinations of base materials and filter materials, fifteen set of experiments were done, in these experiments three types of base materials and five types of filter materials were used. Particle size distribution (PSD) curves of base and filter materials and the constriction size distribution (CSD) curve of the filter medium were plotted for each combination. 


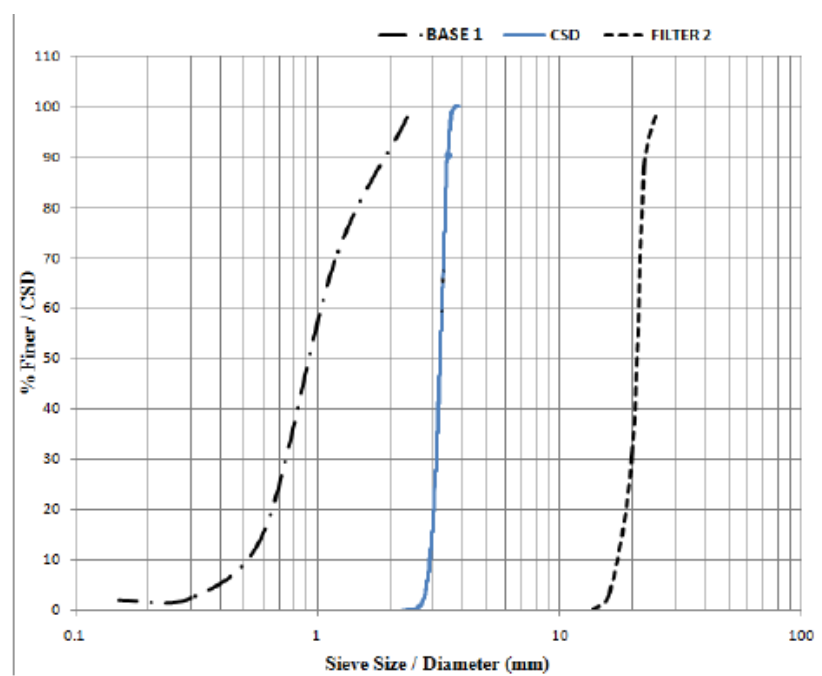

Figure 4 CSD curve of filter materials and PSD curve of base and filter materials.

\section{RESULTS AND DISCUSSION}

\subsection{Terzaghi's criteria}

The current design practice for filters in earth and rock fill dams is an outgrowth of a wellknown concept proposed by Terzaghi. His simple empirical criteria were; $\mathrm{D}_{15} / \mathrm{d}_{85}<5$ for hydraulic stability (retention criterion) and $\mathrm{D}_{15} / \mathrm{d}_{15}>5$ for permeability (non-clogging).[3]

Table1 shows the observations from the fifteen experiments conducted with different combinations of base maerial and filter material. Out of the fifteen experiments, three of the filters failed and the remaining twelve were found to be effective in controlling the erosion of base material. Among these twelve effective filters, only four of the filters have the ratio $\mathrm{D}_{15} / \mathrm{d}_{85}<5$ and the remaining have the value greater than 5 . But all the failed filters have the ratio greater than 5. Also, no clogging of filters was observed. As expected,the Terzaghi's criterion is conservative for uniform base soil because those filters which have the ratio $D_{15} / d_{85}$ greater than 5 did not fail upto a value of the ratio nearly 18 . This also indicates that there is a wide selection of filters for a given base soil, making it difficult to arrive at the most suitable one with Terzhagi's criterion. Hence one more criterion, which is the constriction size distribution, is introduced to narrow down the selection of a filter.

Table 1 Experiments as per Terzagi criteria

\begin{tabular}{|c|c|c|c|c|}
\hline Exp.No & $\mathbf{C}_{\mathbf{u}}$ Filter & $\mathbf{C}_{\mathbf{u}}$ Base soil & $\mathbf{D}_{\mathbf{1 5}} / \mathbf{d} \mathbf{8 5}$ & $\begin{array}{c}\text { Experimental } \\
\text { Results }\end{array}$ \\
\hline 1 & 1.36 & 2 & 3.35 & Effective \\
\hline 2 & 1.36 & 3.3 & 8.14 & Effective \\
\hline 3 & 1.36 & 4.5 & 3.17 & Effective \\
\hline 4 & 1.03 & 3.3 & 10.59 & Effective \\
\hline 5 & 1.03 & 3.3 & 25.71 & Failed \\
\hline 6 & 1.03 & 4.5 & 10 & Effective \\
\hline 7 & 1.04 & 2 & 7.65 & Effective \\
\hline 8 & 1.04 & 3.3 & 18.57 & Failed \\
\hline 9 & 1.04 & 4.5 & 7.22 & Effective \\
\hline 10 & 1.58 & 2 & 3.59 & Effective \\
\hline 11 & 1.58 & 3.3 & 8.71 & Effective \\
\hline 12 & 1.58 & 4.5 & 3.39 & Effective \\
\hline 13 & 1.07 & 2 & 7.82 & Effective \\
\hline 14 & 1.07 & 3.3 & 19 & Failed \\
\hline 15 & 1.07 & 4.5 & 7.39 & Effective \\
\hline
\end{tabular}




\subsection{Constriction Size Distribution Criterion}

Constriction size distribution curves were plotted for five filter materials. By taking different ratios of constriction size from CSD curve of filter materials and percentage finer from PSD curve of base materials, it was found that when the ratio $\mathrm{C}_{10} / \mathrm{d}_{95}<2$, the filter was effective in controlling the erosion ; $\mathrm{C}_{10}$ being $10 \%$ constriction size distribution from CSD curve of filter material .

Table 2 Experimental results as per constriction size distribution criterion

\begin{tabular}{|c|c|c|c|c|c|}
\hline Exp No & $\mathbf{C}_{\mathbf{u}}$ Filter & $\begin{array}{c}\mathbf{C}_{\mathbf{u}} \text { Base } \\
\text { soil }\end{array}$ & $\mathbf{C}_{\mathbf{1 0}} / \mathbf{d}$ 95 & Result & $\begin{array}{c}\text { \% by weight } \\
\text { of Particles } \\
\text { eroded }\end{array}$ \\
\hline 1 & 1.36 & 2 & 0.46 & Effective & Nil \\
\hline 2 & 1.36 & 3.3 & 1.12 & Effective & 0.12 \\
\hline 3 & 1.36 & 4.5 & 0.43 & Effective & Nil \\
\hline 4 & 1.03 & 3.3 & 1.32 & Effective & 2.14 \\
\hline 5 & 1.03 & 3.3 & 3.23 & Failed & 100 \\
\hline 6 & 1.03 & 4.5 & 1.23 & Effective & 0.25 \\
\hline 7 & 1.04 & 2 & 0.88 & Effective & 0.10 \\
\hline 8 & 1.04 & 3.3 & 2.15 & Failed & 40.27 \\
\hline 9 & 1.04 & 4.5 & 0.82 & Effective & 0.07 \\
\hline 10 & 1.58 & 2 & 0.49 & Effective & Nil \\
\hline 11 & 1.58 & 3.3 & 1.21 & Effective & 0.13 \\
\hline 12 & 1.58 & 4.5 & 0.46 & Effective & Nil \\
\hline 13 & 1.07 & 2 & 1.06 & Effective & 0.12 \\
\hline 14 & 1.07 & 3.3 & 2.60 & Failed & 100 \\
\hline 15 & 1.07 & 4.5 & 0.99 & Effective & 0.11 \\
\hline
\end{tabular}

If $\mathrm{C}_{10} / \mathrm{d}_{95} \leq 2$, it will be an effective filter. This ratio explains the behavior of filters more clearly. The results in Table 2 which is based on fifteen combinations, show that the erosion of base material increases with the increase in the $\mathrm{C}_{10} / \mathrm{d}_{95}$ ratio. With just one parameter $\mathrm{D}_{15}$ of the filter, the choice of filters are many, and as per the Terzaghi's criterion, any of these filters can be chosen. This will impose difficulties to choose the best among the many and since the constriction size distribution of all these filters are different, the selection of the most suitable filter is made more easy..

\section{CONCLUSIONS}

Terzaghi's criterion $\left(D_{15} / d_{85}<5\right)$ is not found to be valid in all cases. However it is conservative. Constriction size distribution criterion which relates the voids in the filter to the particle size of the base material is a better approach to study the behaviour of filters, than the Terzaghi's criterion $\left(D_{15} / d_{85}\right)$, which is based on particle size alone. Filtration mechanisms take place in the void network of the filter material and particle infiltration is controlled by constriction sizes. The proposed constriction size based criterion, $C_{10} / d_{95} \leq 2$. directly compares the controlling constriction size $\left(C_{10}\right)$ with the specific representative base size (d d95) to indicate the effectiveness of filters. When $C_{10} / d_{95}$ increases the particle eroded is also found to increase. $C_{10} / d_{95}>2$ defines the ineffectiveness of filters, this ratio explains the behaviour of filters rationally with respect to the constriction sizes and provides a better criterion to select a filter for a given base material, for controlling the erosion. 


\section{REFERENCES}

[1] Budhima Indraratna, Ashok K Raut and Hadi Khabbaz, "Constriction - Based Retention Criterion for Granular Filter Design" Journal of Geotechnical and Geo Environmental Engineering, ASCE, 2007,113,266-276

[2] Jonathan Fannin. "Karl Terzaghi: From Theory to Practice in Geotechnical Filter Design". Journal of Geotechnical and Geo environmental Engineering, ASCE, 2008,134,267-276

[3] K. Arunlanandan, Edward B. Perry. "Erosion in Relation to Filter Design Criteria in Earth Dams". Journal Of Geotechnical Engineering, ASCE 1983,109.682-698 M. A. Lone,B. Hussain, G. L. Asawa. "Filter Design Criteria for Graded Cohesionless Bases". Journal of Geotechnical and Geoenvironmental Engineering ASCE, 2005,131, 251-259.

[4] N. Reboul, E. Vincens, B. Cambou. "A Computational Procedure to Assess the Distribution of Constriction Sizes for an Assembly of Spheres". Computers and Geotechnics 2010,37,195-206

[5] United States Department of Agriculture, Natural Resources Conservation Service. "Gradation Design of Sand and Gravel Filters". Part 633 National Engineering Handbook, October 1994

[6] Yantao Cui, John K. Wooster, Peter F. Baker, Scott R. Dusterhoff, Leonard S. Sklar, William E. Dietrich". Theory of Fine Sediment Infiltration into Immobile Gravel Bed". Journal of Hydraulic Engineering, ASCE ,2008,134,1421-1429

[7] Yu-Hua Zou, Qun Chen, Xiao-Qing Chen, Peng Cui. "Discrete Numerical Modeling of Particle Transport in Granular Filters". Computers and Geotechnics 47 (2013) 48-56.

[8] Mohammed Y. Fattah, Salama N. Y. Al-Labban and Firas A. Salman, Seepage Analysis of a Zoned Earth Dam by Finite Elements, International Journal of Civil Engineering and Technology (IJCIET), Volume 5, Issue 8, August (2014), pp. 128-139

[9] Dr. S. P. Tatewar and Laxman N. Pawade, Stability Analysis of Earth Dam by Geostudio Software, International Journal of Civil Engineering and Technology (IJCIET), Volume 3, Issue 2, July- December (2012), pp. 437-446 